\title{
Zwischen Schonraum und Krisenherd - Psychologische Beratung für Studierende an der Pädagogischen Hochschule Zürich
}

\author{
Hanspeter Mathys (Zürich)
}

\begin{abstract}
Zusammenfassung: Welche spezifischen Herausforderungen bringt das Studium an einer Hochschule mit sich und was könnte das heissen für die psychologische Beratungsarbeit, die von einem psychoanalytischen Ansatz ausgeht? Am Beispiel der Psychologischen Beratungsstelle an der Pädagogischen Hochschule Zürich werden diese Fragen diskutiert. Dabei geht es vor allem um eine Haltung, die sich einerseits von anderen Beratungsverständnissen, anderseits vom psychoanalytischen Arbeiten im therapeutischen Setting unterscheidet. Der Text versteht sich als subjektiver Erfahrungsbericht, ohne den Anspruch aufeinen wissenschaftlich fundierten State of the Art erheben zu wollen.
\end{abstract}

Schlüsselwörter: Psychoanalytische Beratung, Hochschule, Erstgespräch, Containment

Die psychosoziale Situation von Studierenden an Hochschulen ist zwiespältig. Einerseits bildet die Hochschule einen gewissen Schonraum. Die Zeit des Studiums ist von einer relativen Freiheit geprägt. Es existiert meist noch kein Druck, den eigenen Lebensunterhalt bestreiten zu müssen, und die Arbeit Studierender hat meist nur Konsequenzen für sie selbst. Anderseits bildet die Hochschule ein neues Sozialisationsfeld und somit eine potenzielle Krisensituation. Man ist gefordert, sich in einem verhältnismässig anonymen Umfeld zurecht zu finden, sich einen akademischen Arbeitsstil mit einem gewissen Mass an Selbstverantwortung und Eigeninitiative anzueignen. Eventuell ist auch ein Auszug aus dem Elternhaus und ein Wohnortswechsel mit dem Studium verbunden.

Das Befinden von Studierenden und allfällige psychische Belastungen fanden im deutschsprachigen Raum Anfang der 1970er-Jahre verstärkte Aufmerksamkeit. Von den Hochschulen wurden damals psychologische Beratungsstellen und psychotherapeutische Behandlungszentren gegründet. An der Pädagogischen 
Hochschule Zürich (PHZH) können sich Studierende seit 2002 unentgeltlich psychologisch beraten lassen.

\section{Rahmenbedingungen}

Seit fünf Jahren leite ich diese Stelle, und ich war gespannt darauf, wie es gelingt, eine psychoanalytische Haltung auf ein Beratungssetting anzuwenden. Nicht nur der Auftrag und die Dauer der Behandlung unterscheiden sich grundlegend vom psychotherapeutischen und psychoanalytischen Setting. Auch das innenarchitektonische Schibboleth der Psychoanalyse, die Couch, gibt es nicht in meinem Beratungsraum. Dafür stehen da zwei Sessel und ein rundes Tischchen dazwischen. Das ist schon ein ziemlicher Unterschied zum Alltag der PH-Studierenden, die den Grossteil ihres Studienalltags an Schulbänken sitzend verbringen. So merken sie schon beim Eintreten, dass hier etwas anders läuft, als sie es gewohnt sind.

Die Arbeitsbedingungen sind gediegen. Wir haben zehn Sitzungen pro Person zur Verfügung, das ist viel im Vergleich mit anderen Hochschulen. Die Arbeit in einer Institution des Bildungswesens ist mit derjenigen in medizinisch dominierten Kliniken nicht vergleichbar. Ich habe viel Freiheit, kann, darf und muss in kürzester Zeit eine Einschätzung, manchmal auch eine Diagnose stellen sowie eine Therapieindikation verantworten, obwohl ich als Psychologe das ja gar nicht darf und kann. Jedenfalls wenn es nach der Meinung einiger Psychiater geht, die diese Kompetenzen quasi als Alleinstellungsmerkmal für sich reklamieren und sich damit weiterhin den Zugang zum Futtertrog der Grundversicherung sichern wollen (so etwa Seifritz, 2019). Es gibt auch weit und breit keine Krankenkasse, die aufgrund eines vertrauensärztlichen Gutachtens, das unter Umständen von einem Hals-, Nasen-, Ohrenarzt erstellt wurde, die weitere Behandlungstranche absegnet oder eben auch nicht.

Ein Nachteil hingegen ist die fehlende Kultur der Schweigepflicht. Wenn mich beispielsweise Dozierende in der Mensa mal so quer über das Salatbuffet ansprechen, sie hätten die Frau Müller zu mir in die Beratung geschickt und ob sie schon angekommen sei, dann muss ich die Kolleginnen und Kollegen in Schranken weisen und immer wieder betonen, dass die Schweigepflicht das A und O unserer Arbeit ist und falls je ein Verstoss dagegen ruchbar würde, wir unseren Laden dichtmachen könnten. Ebenso musste ich zu Beginn dafür kämpfen, dass ich nur Ratsuchende, die auf freiwilliger Basis in die Beratung kommen, beraten werde. Davor konnten Studierende, bei denen die Frage im Raum stand, ob sie geeignet seien, den Lehrberuf auszuüben, verpflichtet werden, die psychologische Beratung 
aufzusuchen. Beratung als pädagogische Auflage, wenn nicht sogar als Strafaufgabe ist keine besonders günstige Ausgangslage. Und spätestens seit Argelander (1970) weiss man, dass der «geschickte» Patient gar nicht anders kann, als sich tendenziell ungeschickt anzustellen.

Abgesehen von solchen fachkulturellen Differenzen habe ich den Eindruck, die Beratungsarbeit werde von den meisten Kolleginnen und Kollegen der PH geschätzt und respektiert. Das ist nicht zuletzt deshalb wichtig, weil ich auf sie angewiesen bin, sie sozusagen meine «Zuweiser« sind. Wenn Mentorinnen und Mentoren, die wichtigsten Bezugspersonen der Studierenden, oder Dozierende merken, dass jemand in Schwierigkeiten ist, dann können sie auf unsere Stelle aufmerksam machen. Und umgekehrt kann ich der Institution berichten, was mir auffällt, wo sich vielleicht problematische Entwicklungen verdichten, die nicht nur individuell, sondern institutionell angegangen werden sollten.

Die Anliegen, mit denen Studierende in die Beratung kommen, sind sehr unterschiedlich und können grob in zwei Gruppen eingeteilt werden: einerseits Fragen, die unmittelbar im Zusammenhang mit dem Studium stehen, anderseits private Probleme, die aber meist auch Folgen für das Studieren haben. Die Studienthemen zeichnen sich oft durch eine gewisse Dringlichkeit aus. Wenn Prüfungen bevorstehen und die Studierenden von Prüfungsangst geplagt sind oder Arbeiten schon längst hätten abgegeben werden sollen, was aber nicht termingerecht gelang, komme ich mir bei der Beratung nicht besonders psychoanalytisch vor, sondern eher als Krisenmanager. Manchmal sind es recht dramatische Situationen, etwa dann, wenn Studierende in einen Teufelskreis aus eigenem Versagensgefühl, Scham und Vermeidung geraten und seit einem halben Jahr keinerlei Brief- oder Elektropost mehr geöffnet haben.

Nicht selten taucht die Frage auf, ob dieses Studium das Richtige ist und ob der Lehrberuf der passende ist und überhaupt die Frage, wer und was zu mir passt und was nicht, mit anderen Worten Fragen der Identitätsbildung, die fast schon regelmässig an Fragen der Ablösung vom Elternhaus gekoppelt sind. Nicht selten sind dies relativ schmerzliche Prozesse für die Ratsuchenden: Indem sie ihre eigene Welt erschaffen, müssen sie auf die Zustimmung und Anerkennung der Eltern verzichten. Vermeiden sie dies, bleiben sie latent oder manifest in einer psychischen Position gefangen, die darauf ausgerichtet ist, es den Eltern recht zu machen und die adoleszente Attacke zu vermeiden. Wo dies der Fall ist, können regressive Lösungen dominant bleiben und in eine adoleszente Depression führen. Der depressive Heranwachsende ist dann ständig vom Wunsch beseelt, der Idealvorstellung seiner Eltern gerecht zu werden - nur um schliesslich festzustellen, 
dass er dazu nicht imstande ist oder nur zum Preis des Verzichts auf ein eigenes Leben (King, 2012).

Mit dieser kleinen Expedition ins Reich der Identitätsfindung wird deutlich, dass es sich mehrheitlich um Ratsuchende handelt, die sich im weitesten Sinne in einer spätadoleszenten Phase befinden. Aber nicht nur: auch Studierende, die als QuereinsteigerInnen die Ausbildung absolvieren, kommen in die Beratung, Personen also, die schon eine Ausbildung abgeschlossen haben und mitten im Leben stehen, oft mit Familie, eigenen Kindern und all den ganz anderen sozioökonomischen Herausforderungen als etwa der 19-jährige Student, der noch zuhause bei den Eltern wohnt. Nicht zuletzt diese Altersspanne des Klientels von etwa 19-59 Jahren macht die Beratungsarbeit abwechslungsreich.

\section{Erstgespräche}

Meine Arbeit besteht zu einem grossen Teil aus Erstgesprächen. Früher waren das die Situationen, die mir oft etwas Unbehagen bereiteten. Mittlerweile überwiegt die Neugierde. Ich bin gespannt, wer kommt, wie die Begrüssung abläuft, ob die Studierenden gleich zur Sache kommen wollen, bevor ich meine formale Eingangsrede mit der Schweigepflicht machen kann, welche Anliegen sie mitbringen und wie sie diese in Worte fassen und ob sie mit dem, was ich ihnen mitteile, etwas anfangen können. Wenn Studierende das erste Mal in die Beratung kommen, ist die entsprechende Gemütsverfassung immer die einer mehr oder weniger ausgeprägten Ambivalenz. Grundsätzlich wollen sie mit ihren Schwierigkeiten allein zurechtkommen. Mit dem Schritt über die Schwelle in den Beratungsraum respektive mit der Anmeldung ist das Zugeständnis verbunden, dass dies nicht mehr möglich ist. Das ist kränkend. Und dieser Tatsache versuche ich Rechnung zu tragen, aber immer nur implizit, nicht explizit. Ich würde nie sagen: «Toll, dass Sie es geschafft haben, sich hier anzumelden!» Wenn das jemand zu mir sagen würde in einer solchen Situation, wäre das ein Grund, aufzustehen und das Weite zu suchen. Vielmehr versuche ich eine Gesprächsatmosphäre herzustellen, die von einer gewissen Selbstverständlichkeit geprägt ist. Das heisst, ich bin aufmerksam, versuche, gut zuzuhören, und versuche vor allem, eine Atmosphäre zu schaffen, in denen sich die Studierenden sicher fühlen. Ich glaube, die Ratsuchenden, die sich meist in einer unsicheren Situation befinden, suchen in erster Linie ein sicheres Gegenüber. Das Gefühl von Sicherheit kommt meines Erachtens am ehesten zustande, wenn Ratsuchende merken, der Berater kann auch in einer emotional belastenden Situation klar denken und eine eigene Perspektive einnehmen. Wenn ich Bions Vorstellung vom «Containment» richtig verstehe, geht es ihm ganz 
zentral um diesen Punkt, und ich sehe es als meine Verantwortung an, meine Denkfähigkeit aufrechtzuerhalten oder wiederherstellen zu können, wenn sie mir abhandenkommt. Dazu braucht es eine gewisse Distanz zu den Ratsuchenden, die einem "fremden» Berater von seiner Rolle her leichter fällt als beispielsweise den Eltern. Diese sind häufig in die Konflikte mit verstrickt und dadurch befangen. Katastrophen ihrer Kinder bedeuten somit meist auch Katastrophen für sie selbst. Auch Freunde und Bekannte reagieren meist so. Viele im privaten Umfeld haben im weitesten Sinne private eigene Interessen in der Beziehung zu Ratsuchenden. Das ist in der Beratung anders. Containment verstehe ich als ein Konzept, das nicht nur für schwer kranke PatientInnen von Bedeutung ist, sondern auch in der Beratungssituation, wo die zentrale Frage darum kreist, was der Ratsuchende mitteilen kann und dadurch eine erste Entlastung erlebt oder eben nicht. Wenn dieses initiale Containment im Erstgespräch gut gelingt, kann etwas sehr Interessantes geschehen: Studierende melden sich manchmal mit einem vermeintlich «harmlosen» Anliegen an. Erst im Verlauf der Beratung zeigt sich dann, dass oft wesentlich gravierendere Schwierigkeiten und Belastungen die Studierenden umtreiben. Wenn sich jemand mit Lernproblemen anmeldet, dient dies manchmal als eine Art «Eintrittsticket» in die Beratung, um im weiteren Verlauf von einer seit geraumer Zeit bestehenden Essstörung zu erzählen. Wenn Erstgespräche einem solchen behutsamen Abtasten gleichen, geht es erst einmal darum, dass Ratsuchende das beratende Gegenüber kennenlernen wollen, und erst dann entscheiden, was mitgeteilt werden kann, wie viel davon und auf welche Art und Weise.

Ich bin immer wieder erstaunt, welche Kompetenzen die Studierenden mitbringen. An der $\mathrm{PH}$ werden sie ordentlich getrimmt auf Selbstreflexion, was ihnen teilweise fast schon aus den Ohren herausquillt. Für die Beratung ist es Gold wert. In Kombination mit einer gut ausgebildeten Introspektionsfähigkeit und Verbalisierungskompetenz ist in kurzer Zeit viel möglich. Oft reichen zwei oder drei Gespräche aus, und vieles kommt wieder in Gang, was vorher blockiert war. Dabei spielt die (oft nur implizite) Ermunterung, einfach mal drauflos zu erzählen, also sich assoziativ zu äussern, ohne Rücksicht auf inhaltliche Stringenz oder gar Struktur eine nicht unerhebliche Rolle. Einige fragen explizit nach, ob sie jetzt tatsächlich so wild herumspringen dürften, wenn ihnen jetzt noch etwas ganz anderes in den Sinn käme.

Nicht selten kommen Studierende, die einen Rat oder ein paar Tipps wollen. Die habe ich selten auf Lager. Das führt manchmal zu Ernüchterung und Enttäuschung. Ich versuche dann den Ratsuchenden zu erklären, dass ich ihre Situation und ihr Anliegen nicht ernst genug nähme, wenn es ein paar einfache 
Tipps gäbe, die ich so aus dem Handgelenk schütteln könnte. Am aufregendsten finde ich es, wenn Studierende mit einer relativ technischen Vorstellung kommen, etwa mit der Frage «Können Sie mir Strategien vermitteln, wie ich mich besser abgrenzen kann?» und es dann im Gespräch möglich wird, eine andere Haltung zu entwickeln; wenn es gelingt, statt etwas Störendes loswerden zu wollen, ein Interesse für das eigene störende Erleben und Verhalten entwickeln zu können und das auszumerzende «Dysfunktionale» in eine Fragestellung umzuwandeln, wieso dieses Dysfunktionale einst sehr funktional war. Das ist für mich der Kern des psychoanalytischen Arbeitens im Rahmen einer kurzen Beratung.

Ich möchte versuchen, dies anhand einer kurzen Fallvignette zu illustrieren:

\section{Fallvignette: Pingpong}

K. berichtet im Erstgespräch von schwierigen Erfahrungen in der Kindheit. DerVater habe die Entwicklung ihres Selbstwertgefühls eher gestört als gefördert. Er habe sie als Tochter abgelehnt und sei abgetaucht, als sie acht Jahre alt war. Seitdem habe sie keinen Kontakt mehr zu ihm. Sie berichtet weiter, dass ein naher Verwandter darauf zu einer Art Vaterersatz wurde. Für ihn seien gute Noten nie gut genug gewesen. Immer habe er gefragt: «Warum nicht besser?»

Heute gehe es ihr gut, im Studium habe sie keine Probleme, es gelinge ihr alles auf Anhieb. Aber in sozialen Beziehungen habe sie das Gefühl, sie genüge nicht und sie sei nur liebenswert, wenn sie perfekt sei. Mit ihrem Freund reize sie das immer wieder aus: sie provoziere ihn bis zu dem Punkt, wo sie «recht» bekommt, dass sie nicht liebenswert sei, wenn sie nicht perfekt sei. Dagegen wünscht sie sich Strategien.

Ich teile ihr meine Einschätzung mit: das 8-jährige Mädchen sei mit dem Vater noch nicht fertig, sie suche die Auseinandersetzung und eine bessere Entwicklung. Dadurch verstricke sie sich in Auseinandersetzungen mit ihrem Freund. Es gehe weniger ums Abklemmen, um Strategien gegen irgendetwas, sondern darum, wie sie den Fluss dieser psychischen Entwicklung so kanalisieren könne, dass daraus weniger destruktive Auswirkungen für ihre aktuellen Beziehungen entstehen.

In einer der folgenden Sitzungen liest sie dann (auf Anregung aus einer früheren Sitzung) eine Art Dialog zwischen zwei Figuren vor. Aber es ist gar kein richtiger Dialog, es ist eher so, dass die erste Stimme etwas sagt, z. B. «Alle Menschen sind liebenswert» und die zweite etwas antwortet, z. B. «Ja schon, aber diejenigen, die mehr leisten, sind wertvoller». Der Dialog findet nur in eine Richtung statt. Als ich das sage, fällt ihr «Pingpong» ein, und wir merken, dass es ein Pingpong- 
spiel ist, bei dem die eine Seite immer anspielt und die andere den Ball so zurückspielt, dass er von der anspielenden Seite nicht mehr erreicht werden kann. Der Ballwechsel endet sozusagen mit dem Return, die retournierende Seite macht den Punkt, sodass die erste immer wieder neu anspielen muss. Der Ball bleibt nicht im Spiel. Die zweite Seite dominiert das Spiel und gewinnt klar. Es scheint, dass die rigide und fordernde Seite die bestimmende ist. Das ist aber noch nicht alles. Dadurch, dass K. dieses «Pro-und-Kontra-Pingpong» mündlich schildert, streut sie immer wieder Kommentare ein, ergänzt beispielsweise, dass sie jetzt diese Aussage etwas schroff findet, positioniert sich dadurch selber neu und setzt den abgebrochenen inneren Dialog so fort. Es entsteht eine Art dritte Instanz, die nun in einem Moment der Nachträglichkeit in der Lage, aber auch gefordert ist, das ungleiche Spiel dieser beiden internalisierten Objektrepräsentanzen neu zu moderieren und zu modulieren.

Neben einer Art Umformulierung der Problemstellung wird im zweiten Teil dieser kurzen Fallvignette auch etwas vom Konzept der gleichschwebenden Aufmerksamkeit deutlich. Das Aufgreifen des spontanen Einfalls «Pingpong» sowie die genaue und ausführliche Betrachtung, wie spezifisch $i h r$ Spiel abläuft, stellt eine Perspektive dar, die von der Alltagskommunikation und wahrscheinlich auch von anderen Beratungsansätzen abweicht und dadurch möglicherweise etwas Neues beinhaltet.

Dieser letzte Punkt zeichnet meines Erachtens ein psychoanalytisch geprägtes Erstgespräch aus, weil das Neue nichts anderes als das «Noch nicht bewusst Zugängliche» ist. Sowohl in der allgemeinen Beratungsliteratur als auch von der Erwartungshaltung der Ratsuchenden aus betrachtet, ist die Vorstellung darüber, was einen umtreibt und in ein Erstgespräch führt, von einer ziemlich programmatischen Auffassung geprägt. Im Vordergrund steht der bewusstseinsfähige Aspekt und ein höherer Grad an Ausarbeitung einer Problemstellung, als dies in der Realität eines Erstgesprächs der Fall ist. Aus psychoanalytischer Sicht kommen dabei zwei Dimensionen zu kurz: einerseits die Dimension des Unbewussten, und anderseits die Tatsache, dass Ratsuchende ein Gegenüber aufsuchen, an das sie ihr Problem adressieren, also der Beziehungsaspekt im Erstgespräch. Ich versuche, dies zu betonen, indem ich bei der Einleitung nie frage, was das Problem ist, sondern was das Anliegen der Ratsuchenden ist. Ein persönliches Anliegen wird an ein Gegenüber herangetragen und beinhaltet mehr als eine nüchterne Problemstellung. Das Herausfordernde besteht nun darin, dass dieser nicht bewusst zugängliche Teil des Anliegens gar nicht ausgesprochen werden kann. Das Anliegen gestaltet sich vielmehr in der Inszenierung der Begegnung. In dieser Form 
zielt es darauf ab, Beteiligung und nachvollziehende Resonanz beim Gegenüber zu entwickeln, und zwar nicht per Thematisierung, sondern im Vollzug des Gesprächs (zum Konzept des Anliegens vgl. Mathys et al., 2013).

\section{Triage}

Eine weitere Herausforderung der Beratungsgespräche besteht darin, relativ rasch zu beurteilen, ob eine Beratung hier sinnvoll ist oder ob eine Psychotherapie angezeigt ist. Wenn letzteres der Fall ist, geht es darum, relativ zügig eine Überweisung in die Wege zu leiten. Bei etwa einem Fünftel aller Ratsuchenden ist eine Psychotherapie indiziert. Wie bei den meisten Hochschulambulanzen auch handelt es sich vorwiegend um PatientInnen mit einer Angststörung und/oder depressiven Episoden.

Bei der Therapievermittlung muss ich mir die Frage stellen: Wer passt am besten zu wem? Diese Wahl läuft in erster Linie vorbewusst ab. Es kommt vor, dass schon während des Erstgesprächs klar wird, dass ich jemandem eine Psychotherapie empfehlen werde, und es taucht bereits ein Gesicht einer Kollegin, eines Kollegen auf, die oder den ich empfehlen werde. Manchmal wird mir dann klarer, wieso sich gerade diese Person aufgedrängt hat. Auch hier kommt wieder das Prinzip der Nachträglichkeit ins Spiel.

Bei der Überweisung ermutige die Studierenden, kritisch zu sein, sich nicht mit der erstbesten Adresse, die ich ihnen vermittle, zufrieden zu geben. Das führt manchmal zu erstaunten Reaktionen: «Darf ich denn das, jemanden ablehnen?» Manchmal sind die Überweisungen auch schwierig, meist dann, wenn die Beratungen schon etwas länger dauern und eine Bindung entstanden ist. Da fällt es den Ratsuchenden manchmal schwer, jemand andern aufzusuchen, was sich daran zeigt, dass sie fragen, ob sie dann diesem/r anderen die ganze Geschichte nochmals von vorne erzählen müssen oder ob nicht ich dies für sie tun könnte. Ja und nein lautet die Antwort. Ja, sie müssen die ganze Geschichte nochmals erzählen. Und nein, das kann nicht ich übernehmen. Es zeigt sich hier nochmals die oben beschriebene Ausgangslage beim Erstgespräch: Es geht eben nicht um pure Informationsvermittlung, sondern auch darum, im Gespräch das Gegenüber kennenzulernen, um abschätzen zu können, ob man hier nun am richtigen Platz ist, um eine Psychotherapie beginnen zu können. Manchmal fällt es auch mir als Berater nicht so leicht, jemanden gehen zu lassen, weil sich ein gutes Arbeitsbündnis entwickelt hat und ich am liebsten selber weiterarbeiten würde.

Bei Therapieempfehlungen bin ich zuweilen nicht besonders neutral, sondern etwas suggestiv. Ich sage den Studierenden dann, dass ich ihnen eine 
Psychotherapie sehr ans Herz lege und ich den Eindruck habe, sie könnten davon profitieren. Ich sage das oft mit Nachdruck und nicht, als wäre es mir gleichgültig, wie sie sich entscheiden würden. An diesem Punkt unterscheide ich zwischen Beratung und Psychotherapie/Psychoanalyse. Ich beziehe in der Beratung oft Stellung. Wenn beispielweise ein Student zu mir kommt und unsicher ist, ob dieses Studium das Richtige für ihn ist und der Lehrerberuf passt, sage ich manchmal, nachdem ich mir alle Gedanken angehört habe: «Also ich an Ihrer Stelle würde jetzt Folgendes machen....., aus diesem und jenem Grund...., aber ich weiss nicht, ob das auch für Sie das Passende wäre.» So etwas würde ich in der Analyse oder der Therapie nie machen.

Stimmt nicht ganz. Einmal habe ich es genauso gemacht. Die Reaktion der Analysandin auf der Couch war bemerkenswert. Sie zuckte regelrecht zusammen und rief: «Was???! Das haben Sie ja noch nie gemacht!» Und seitdem habe ich es auch nie mehr gemacht.

\section{Scheitern}

Im Allgemeinen erlebe ich die Studierenden, die in die Beratung kommen, als beflissen und angepasst. Sie wollen es ganz besonders gut machen. Erstaunt bin ich immer wieder, wenn 20-Jährige mir gegenübersitzen, bereits ein Semester an der Uni waren und jetzt an der PH mit Panik-geweiteten Pupillen berichten, sie seien jetzt Anfang 20 und hätten noch immer keine Ausbildung. Anfang 20 und noch immer keine Ausbildung? Da würde ich am liebsten (und manchmal tu ich's auch!) väterlich milde lächeln und sagen: «Nun entspannen Sie sich mal, ich musste 32 Jahre alt werden, bis ich endlich wusste, was ich will. Davor habe ich einige Jahre damit verbracht, ohne inneren Kompass an Hochschulen herumzugurken und so zu tun, als wär's das jetzt.» Aber wenn ich so rede, nützt es nichts. Ich bin es dann, der ein gequältes Lächeln erwidert bekommt. Einfach mal so anzufangen und keinen Plan zu haben, etwas auszuprobieren und Erfahrungen zu machen, und dann Schlüsse daraus zu ziehen, ist im durchökonomisierten Bologna-Universum ganz offensichtlich nicht vorgesehen. Wer ein Studium abbricht, ist gescheitert, wer eine Prüfung nicht besteht, ein Loser und wer einen Lebenslauf, nein, ein CV, vorweist, der irgendwo auf etwas nicht Geradliniges verweist, kann gleich zum Sozialamt gehen. Etwa so sieht die berufliche Perspektive eines 20-Jährigen aus, und ich bin je länger je weniger sicher, ob das neurotisch ist oder eine verheerende neoliberale Realität, die schon derart internalisiert ist, dass sie den Betreffenden gar nicht mehr auffällt. Alle Studien zeigen das gleiche Bild: An Hochschulen haben Prüfungsängste massiv zugenommen. Das Gefühl zu versagen, ist flächendeckend 
vorhanden, und wenn jemand an Hochschulen das Wort «scheitern» in den Mund nimmt, wird er wohl bald von der Hochschulleitung zurechtgewiesen, mit dem Hinweis, dass ein Wort, das derart traumatisierendes Potenzial in sich berge, am Campus nicht mehr verwendet werden darf.

\section{Infantilisierung}

Gerade an Hochschulen scheint das Phänomen «Trauma» Hochkonjunktur zu haben. Der britische Soziologe Frank Furedi (2017) hat in einer eindrücklichen Studie über die «Infantilisierung der Hochschulen» die wohl kritischste Perspektive auf die Einrichtung psychologischer Beratungsstellen an Hochschulen verfasst. So scheint die Tendenz an angelsächsischen Hochschulen von einer grossen Empfindlichkeit (oder eben Kindlichkeit) und Unfähigkeit zur Auseinandersetzung mit verschiedenen Meinungen, Positionen und Inhalten geprägt zu sein. Furedi stellt in seinem Buch mehrere Szenen zusammen, in denen es zu internen Verfahren gegen Dozierende kam, weil diese den Studierenden schwierige Inhalte zumuteten, mit dem Ziel, dass sich diese damit auseinandersetzten, also beispielsweise mit der Geschichte des Rassismus in den USA. Die Studierenden jedoch nahmen dies nicht als Angebot für ihre eigene Auseinandersetzung mit einer gesellschaftlichen Realität auf, mit der Aussicht, etwas besser zu verstehen und vielleicht ja dann mal als EntscheidungsträgerInnen Verantwortung zu übernehmen, um rassistischen Tendenzen etwas entgegensetzen zu können. Nein, diese Studierenden fühlten sich traumatisiert durch solche Inhalte, beschwerten sich bei der Hochschulleitung und forderten eine Verfügung gegen Dozierende, die ihnen solche schwer verdaulichen Inhalte zumuteten. Und sie bekamen Recht! Nun kommen die psychologischen Beratungsstellen ins Spiel: Diese sollen dann den Studierenden helfen, ihr erlittenes «Trauma» zu verarbeiten - und tun es gemäss Furedi auch. Dass hier offenbar eine akademische Elite heranwächst, die immer weniger erträgt und auch keine Haltung mehr entwickeln kann, wie schwierige Themen zu bearbeiten sind, geistig und psychisch, ist das eine. Dass mit dieser inflationären Reklamation eine Aushöhlung des Traumakonzepts einhergeht und Studierende mit deutlich gravierenderen Traumatisierungen mit denjenigen, die Unangenehmes mit Traumatischem verwechseln, um Behandlungsplätze ringen müssen, ist ein weiteres Ärgernis dieser «Pseudo-Traumawelle». Und es drängen sich einige beklemmende Fragen auf: Tragen psychologische Beratungsstellen dazu bei, unangenehme Alltagserfahrungen zu pathologisieren und als etwas Behandlungsbedürftiges zu verkaufen statt als Lebenserfahrung? Schaffen sie ein Klima, das «Lernen durch Erfahrung» (Bion) mehr sabotiert als fördert? 
Bislang kenne ich solche Verhältnisse nur aus der Literatur, nicht aus der eigenen Beratungsarbeit. Aber die Vorstellung, dass psychologische Beratung Entwicklung zu vermeiden hilft, statt diese zu fördern, ist abschreckend. So wichtig zu Beginn die Vermittlung von Sicherheit in einer unsicheren Situation ist, so wichtig ist auf dem eben dargestellten Hintergrund die ergänzende Haltung, ratsuchenden Studierenden auch etwas zuzumuten, oder anders formuliert, sie arbeiten zu lassen und nicht der Versuchung zu verfallen, ihre Probleme lösen zu wollen. Das würde zwar Grössenfantasien des Beraters fördern aber auch eine infantileVersorgungshaltung bei den Studierenden. Diese dann in mehreren Jahren durchzuarbeiten, dazu fehlt, anders als im psychoanalytischen Setting, die Zeit. Beratung sollte deshalb von Beginn weg progressiv orientiert sein und nicht regressive Prozesse fördern.

\section{Fazit}

Die Ausgangslage von Ratsuchenden ist meist von einer mehr oder weniger ausgeprägten Destabilisierung, Verunsicherung, Hilflosigkeit und Verzweiflung geprägt. Die implizite Erwartungshaltung der Ratsuchenden an die Beratenden lautet infolgedessen: Hilf mir, tu etwas, ich kann nicht mehr, nimm mir die Probleme weg! In der Beratung muss es zu einer Frustration dieser impliziten Erwartungshaltung kommen. Die Ratsuchenden werden vielmehr auf ihren Akteurstatus und ihre Ressourcen angesprochen. Mit anderen Worten, den Ratsuchenden wird nicht das gegeben, was sie aus einer (kindlichen) Erwartungshaltung erhoffen. Dafür sollten sie etwas anderes vermittelt bekommen: Ein Zutrauen, das ihre (aktuell brachliegenden) Fähigkeiten weckt und die erforderliche Energie mobilisieren kann, welche die eigene Selbstwirksamkeit fördern. Die Haltung des Zutrauens in der Beratung ist nicht zu verwechseln mit gut gemeinten Durchhalteparolen wie «Du schaffst das schon!» oder ähnlich gutem Zureden. Eine Studentin stand vor einer Prüfung unter grossem Druck, weil ihr gesamtes Umfeld ihr immer wieder einzureden versuchte, sie solle sich nicht verrückt machen, sie schaffe das eh. «Nein», meinte sie, «ich will auch denken können: es könnte schief gehen.»

\section{Literatur}

Argelander, H. (1970). Das Erstgespräch in der Psychotherapie. Darmstadt: Wissenschaftlicher Buchverlag.

Bion, W. F. (1992). Lernen durch Erfahrung. Frankfurt a. M.: Suhrkamp.

Furedi, F. (2017). What's happened to the University? A sociological exploration of its infantilization. London, New York: Routledge. 
King, V. (2012). Adoleszente Identitätssuche und Ablösung: Entwicklungsanforderungen und Krisenpotenziale in Generationsbeziehungen. In: B. Grimmer, I. Sammet \& G. Dammann (Hrsg.), Psychotherapie in der Spätadoleszenz. Entwicklungsaufgaben, Störungen, Behandlungsformen, (S. 35-47). Stuttgart: Kohlhammer.

Mathys, H.P., Arboleda, L., Boucsein, V., Frei, M., Hermann, M.-L., Luder, M., Neukom, M. \& Boothe, B. (2013). Alexandra - eine multiperspektivische, qualitative Einzelfallstudie zu Anliegen von PatientInnen im psychodynamischen Erstinterview. In: Forum Qualitative Sozialforschung 14 (2), http://www.qualitative-research.net/index.php/fqs/article/view/ 1894 [18.12.2019].

Seifritz, E. 09.04.2019. Psychotherapie-Petition: schlechtere medizinische Versorgung zu höheren Kosten. Neue Zürcher Zeitung, https://www.nzz.ch/ meinung/psychotherapie-petition-schlechtere-medizinische-versorgungzu-hoeheren-kosten-ld.1468304 [18.12.2019].

\section{Angaben zum Autor}

Hanspeter Mathys, Dr. phil, Studium der Klinischen Psychologie und Psychopathologie an der Universität Zürich (1996-2002). Psychoanalytische Weiterbildung am Psychoanalytischen Seminar Zürich (PSZ) (2002-2008). Klinische Lehr- und Wanderjahre in Münsterlingen und Männedorf (2002-2006). Assistent und Oberassistent an der Abteilung Klinische Psychologie, Psychotherapie und Psychoanalyse (2006-2013). 2009 Promotion an der Universität Zürich zum Thema der kommunikativen und interaktiven Funktionen von Traummitteilungen in der psychoanalytischen Therapie. Seit 2009 in eigener psychoanalytischer Praxis. Seit 2014 Leiter der Psychologischen Beratung für Studierende an der Pädagogischen Hochschule Zürich (PHZH) 